\title{
Evidence for a purifying selection acting on the $\beta$-lactamase locus in epidemic clones of methicillin-resistant Staphylococcus aureus
}

\author{
Catarina Milheiriço ${ }^{1}$, Ana Portelinha ${ }^{2}$, Ludwig Krippahl ${ }^{3}$, Hermínia de Lencastre ${ }^{1,4}$ and Duarte C Oliveira ${ }^{1,2^{*}}$
}

\begin{abstract}
Background: The $\beta$-lactamase (bla) locus, which confers resistance to penicillins only, may control the transcription of $m e c A$, the central element of methicillin resistance, which is embedded in a polymorphic heterelogous chromosomal cassette (the SCCmec element). In order to assess the eventual correlation between bla allotypes and genetic lineages, SCCmec types and/or $\beta$-lactam resistance phenotypes, the allelic variation on the bla locus was evaluated in a representative collection of 54 international epidemic methicillin-resistant Staphylococcus aureus (MRSA) clinical strains and, for comparative purposes, also in 24 diverse methicillin-susceptible S. aureus (MSSA) strains.

Results: Internal fragments of blaZ (the $\beta$-lactamase structural gene) were sequenced for all strains. A subset of strains, representative of blaZ allotypes, was further characterized by sequencing of internal fragments of the blaZ transcriptional regulators, blal and blaR1. Thirteen allotypes for blaZ, nine for blal and 12 for blaR1 were found. In a total of 121 unique single-nucleotide polymorphisms (SNP) detected, no frameshift mutations were identified and only one nonsense mutation within blaZ was found in a MRSA strain. On average, blaZ alleles were more polymorphic among MSSA than in MRSA (14.7 vs 11.4 SNP/allele). Overall, blaR1 was the most polymorphic gene with an average of 24.8 SNP/allele. No correlation could be established between bla allotypes and genetic lineages, SCCmec types and/or $\beta$-lactam resistance phenotypes. In order to estimate the selection pressure acting on the bla locus, the average $d N / d S$ values were computed. In the three genes and in both collections $d N / d S$ ratios were significantly below 1 .

Conclusions: The data strongly suggests the existence of a purifying selection to maintain the bla locus fully functional even on MRSA strains. Although, this is in agreement with the notion that in most clinical MRSA strains mecA gene is under the control of the bla regulatory genes, these findings also suggest that the apparently redundant function of blaZ gene for the MRSA resistant phenotype is still important for these strains. In addition, the data shows that the sensor-inducer blaR1 is the primary target for the accumulation of mutations in the bla locus, presumably to modulate the response to the presence of $\beta$-lactam antibiotic.
\end{abstract}

Keywords: $\beta$-lactamase $\beta$-lactam resistance, allelic variation, MSSA, MRSA, mecA stabilization

\section{Background}

Staphylococcus aureus is a leading cause of nosocomial infections and has recently emerged as a community acquired pathogen [1-3]. S. aureus is also a paradigm of adaptive power to antimicrobial chemotherapy, able to develop resistance to virtually all classes of antibiotics

\footnotetext{
* Correspondence: dco@fct.unl.pt

'Laboratory of Molecular Genetics, Instituto de Tecnologia Química e Biológica, Universidade Nova de Lisboa (ITQB/UNL), Oeiras, Portugal Full list of author information is available at the end of the article
}

[4].The acquisition of resistance to $\beta$-lactam antibiotics is particularly relevant in clinical terms. Although $\beta$-lactams (i.e. penicillin $G$ ) were the first class of large-spectrum antibiotics to be introduced into clinical practice, they are still the most widely used due to their high effectiveness, low cost, ease of delivery and minimal side effects [5].

In response to $\beta$-lactam chemotherapy, S. aureus has sequentially acquired two resistance genes: first blaZ, which codes for a $\beta$-lactamase and confers resistance to

\section{() Biomed Central}


penicillins only, and then $\operatorname{mec} A$, which codes for an extra penicillin-binding protein ( $\mathrm{PBP} 2 \mathrm{a})$ with reduced affinity for virtually all $\beta$-lactams $[6,7]$. The transcription of both resistance genes may be controlled by homologous twocomponent systems consisting on a sensor-inducer (BlaR1 and MecR1) and a repressor (BlaI and MecI). Interestingly, in spite of the cross-resistance to virtually all $\beta$-lactams provided by mecA, the great majority (> 95\%) of contemporary MRSA are still positive for the $\beta$ lactamase locus [8]. Moreover, the regulators of blaZ, BlaR1 and BlaI, can efficiently induce $m e c A$ transcription and, do it faster than the "natural" $m e c A$ regulators, MecR1 and MecI $[9,10]$. In addition, since many MRSA strains do not have functional mecI-mecR1 genes due to polymorphisms in the $m e c A$ regulatory region [11], the $m e c A$ transcription is presumably under the control of the blaI-blaR1 genes only. In line with these observations, the presence of the blaZ locus has been shown to promote $m e c A$ acquisition and stabilization $[12,13]$.

In $S$. aureus, the $\beta$-lactamase genes may be located in a plasmid or mobilized into the chromosome by transposon $\operatorname{Tn} 552$ [14]. In contrast to the diversity of $\beta$-lactamase genes found in gram-negative bacteria, all staphylococcal enzymes studied so far are molecular class A serine $\beta$-lactamases placed in functional group $2 \mathrm{a}$ [8]. The mature form of the enzyme has a molecular mass of $30 \mathrm{kDa}$, contains 257 amino acids, and is secreted extracellularly [15]. In 1965, Richmond proposed the subdivision of staphylococcal $\beta$-lactamases in four serotypes [16], but the structural basis of the distinction between types is still uncertain and no clear relationship between sequence and serotype was found [17]. Interestingly, serotypes were shown to have specific geographic distributions [8], which may suggest a relationship between bla-type and genetic lineage. Recently, Olsen et al have studied the allelic variation of the blaZ gene among several staphylococcal species and 11 BlaZ protein types were identified [14]. The multiplesequence alignment of those sequence types suggest a separate evolution for plasmid- and chromosomallyencoded blaZ and a very low frequency for exchange of the $\beta$-lactamase locus between strains and species.

In evolutionary terms, MRSA may be regarded as a recent sub-branch of the $S$. aureus population which has acquired the heterelogous chromosomal cassette containing the mecA gene - the SCCmec element [18]. Molecular epidemiology studies on large collections of MRSA isolates have clearly shown that MRSA has a strong clonal structure and that very few lineages, defined by specific macro-restriction patterns of chromosomal DNA and/or multi-locus sequence types, account for the great proportion of MRSA infections worldwide $[19,20]$. The clonal structure of MRSA population may result from a "host barrier" for the $m e c A$ acquisition, which restricts the number of acquisitions to few more permissive lineages $[13,21]$ and/or from the clonal expansion of previously highly epidemic (MSSA) lineages, which have acquired the $m e c A$ gene. Recent data based on comparative genomics of MRSA lineages [22-24] supports both mechanisms as it seems that, within the same genetic (epidemic) lineage, SCCmec acquisitions may occur continuously at the local level.

In spite of the several lines of evidence suggesting an important role of the bla locus in the acquisition, stabilization and regulation of the $m e c A$ gene, the variability of bla genes at the sequence level has never been evaluated among pandemic MRSA lineages. The present study was conducted in order to evaluate the allelic variability of $\beta$ lactamase locus in a representative collection of internationally epidemic MRSA clones and also, for comparative purposes, in a diverse collection of methicillin-susceptible $S$. aureus strains (MSSA), in an attempt to make evolutionary correlations between $\beta$-lactamase allotypes and $\beta$-lactam resistance phenotypes (i.e. MRSA vs MSSA), $\mathrm{SCCmec}$ types and/or genetic lineages.

\section{Methods}

\section{Strain collection}

$S$. aureus strains used in the present study are listed in Tables 1 (MRSA) and 2 (MSSA). All strains have been previously assigned to genetic lineages by Pulse-field gel electrophoresis (PFGE), multi-locus sequence typing (MLST) and protein A sequence typing (spa typing). MRSA strains have been additionally characterized in terms of their SCCmec types. The presence of a functional $\beta$-lactamase locus was confirmed by nitrocefin disks (Sigma) for all strains, in the presence and absence of an inducer (oxacillin at $0.05 \mathrm{mg} / \mathrm{L}$ ).

\section{Media and growth conditions}

Strains were grown overnight at $37^{\circ} \mathrm{C}$ on tryptic soy agar or tryptic soy broth under aerobic conditions.

\section{DNA isolation}

Total DNA was prepared using the Wizard genomic DNA preparation kit (Promega, Madison, WI, USA), according to the manufacturer's recommendations, except for the addition of lysostaphin at $0.5 \mathrm{mg} / \mathrm{mL}$ and RNase at $0.3 \mathrm{mg} / \mathrm{mL}$ for the lysis step.

\section{DNA amplification and sequencing}

The allelic variation on the $\beta$-lactamase locus was evaluated by sequencing internal fragments of blaZ and its transcriptional regulators, blaI and blaR1, amplified by PCR. Based on the available sequence at GenBank (accession number: X52734) for Tn552 of S. aureus, three pairs of primers were designed as follows $\left(5^{\prime} \rightarrow\right.$ 3'): blaZ F1, GAT AAG AGA TTT GCC TAT GC; blaZ 
Table 1 Characteristics and bla locus allotypes of the MRSA strains used in this study

\begin{tabular}{|c|c|c|c|c|c|c|c|c|c|}
\hline \multirow[t]{2}{*}{ Clonal complex ${ }^{\text {a) }}$} & \multirow[t]{2}{*}{ MLST (ST) } & \multirow[t]{2}{*}{$\mathrm{SCCmec}$ type } & \multirow[t]{2}{*}{ Strain } & \multirow[t]{2}{*}{ Isolation origin } & \multirow[t]{2}{*}{ Isolation year } & \multicolumn{3}{|c|}{ bla locus alleles } & \multirow[t]{2}{*}{ Ref. } \\
\hline & & & & & & blaz & blal & blaR1 & \\
\hline & 247 & I & E2125 & Denmark & 1964 & 1 & $\mathrm{ND}$ & ND & {$[30,31]$} \\
\hline & 247 & $\mathrm{IA}$ & HPV107 & Portugal & 1992 & 1 & ND & ND & {$[30,32]$} \\
\hline & 247 & $\mathrm{IA}$ & BK1953 & USA & 1995 & 1 & ND & ND & {$[30,33]$} \\
\hline & 250 & । & $\mathrm{COL}$ & UK & 1965 & - & & & [30] \\
\hline & 250 & । & BK793 & Egypt & 1961 & 1 & 1 & 1 & {$[30,34]$} \\
\hline & 250 & IA & PER34 & Spain & 1989 & 1 & ND & ND & {$[30,35]$} \\
\hline & 239 & III & ANS46 & Australia & 1982 & 1 & 1 & 1 & {$[30,36]$} \\
\hline & 239 & IIIA & HU25 & Brazil & 1993 & 1 & ND & ND & {$[30,37]$} \\
\hline & 239 & $\| \mathrm{A}$ & HUSA304 & Hungary & 1993 & 1 & ND & ND & {$[30,38]$} \\
\hline \multirow[t]{18}{*}{8} & 239 & $\| \mathrm{A}$ & BK2421 & USA & 1996 & 1 & ND & ND & {$[30,34]$} \\
\hline & 8 & $\mathrm{IVa}$ & USA300 & USA & 1995-2003 & 8 & 4 & 9 & {$[39,40]$} \\
\hline & 8 & $\mathrm{IVa}$ & USA500 & USA & 1995-2003 & 1 & ND & ND & {$[39,40]$} \\
\hline & 8 & $\mathrm{IVd}$ & BK2529 & USA & 1996 & 1 & ND & ND & {$[30,34,39]$} \\
\hline & 8 & $\mathrm{IVd}$ & Bargll17 & USA & 1996 & 1 & ND & ND & {$[30,39,41]$} \\
\hline & 8 & IVE & AR43/3330.1 & Ireland & $1988-2002$ & 1 & 1 & 1 & [42] \\
\hline & 8 & IVh & GRE120 & Greece & 1993 & 1 & ND & ND & {$[39,43]$} \\
\hline & 72 & $\mathrm{IVa}$ & USA700 & USA & 1995-2003 & 9 & - & 4 & {$[39,40]$} \\
\hline & 254 & $\mathrm{IVh}$ & HAR36 & Greece & 2002 & 1 & 1 & 1 & {$[39,44]$} \\
\hline & 770 & $\mathrm{IVb}$ & 8/6-3P & Chicago & 1996 & 3 & 3 & 6 & [45] \\
\hline & 5 & 1 & HAR21 & Finland & 2002 & 1 & $\mathrm{ND}$ & ND & {$[44,46]$} \\
\hline & 5 & I-VAR & PL72 & Poland & 1991 & 1 & ND & ND & {$[30,47]$} \\
\hline & 5 & $\|$ & N315 & Japan & 1982 & 8 & 4 & 9 & [18] \\
\hline & 5 & $\|$ & $\mathrm{JP1}$ & Japan & 1987 & 8 & 4 & 9 & {$[30,48]$} \\
\hline & 5 & $\|$ & BK2464 & USA & 1996 & 4 & 6 & 2 & {$[30,49]$} \\
\hline & 5 & $\|$ & USA100 & USA & $1995-2003$ & 3 & 3 & 6 & {$[40,46]$} \\
\hline & 5 & $\mathrm{IVa}$ & BM18 & USA & 1989 & 4 & 6 & 2 & {$[30,39,50]$} \\
\hline & 5 & IVa & FFP311 & Portugal & 1996 & 11 & 1 & 7 & {$[39,51]$} \\
\hline \multirow[t]{12}{*}{5} & 5 & $\mathrm{IVa}$ & HSA49 & Portugal & 1993 & 11 & ND & ND & {$[39,51]$} \\
\hline & 5 & IVa & HSA74 & Portugal & 1993 & 5 & 3 & 3 & {$[39,51]$} \\
\hline & 5 & IVC & Q2314 & Dallas & 1996 & 3 & ND & ND & [52] \\
\hline & 5 & IVC & USA800 & USA & $1995-2003$ & 1 & ND & ND & {$[39,40]$} \\
\hline & 5 & IVC & ARG9 & Argentina & 1996 & 11 & 7 & 7 & {$[39,51]$} \\
\hline & 5 & $\mathrm{IVd}$ & JCSC4469 & Japan & 1982 & 1 & 1 & 1 & [53] \\
\hline & 5 & $\mathrm{IVg}$ & M03-68 & Korea & 2003 & 3 & ND & ND & [54] \\
\hline & 5 & $\mathrm{IV}_{\mathrm{NT}}$ & COB3 & Colombia & 1996 & 6 & 5 & 10 & {$[30,39,55]$} \\
\hline & 5 & $\mathrm{Vl}$ & HDE288 & Portugal & 1996 & 10 & - & 5 & {$[51,56]$} \\
\hline & 5 & $\mathrm{Vl}$ & HUC136 & Portugal & 1995 & 10 & - & 5 & {$[51,56]$} \\
\hline & 228 & I & HAR40 & Belgium & 1995 & 2 & 3 & 1 & {$[44,46]$} \\
\hline & 30 & $\mathrm{IVC}$ & DEN2946 & Denmark & 2001 & 1 & 1 & 1 & {$[39,57]$} \\
\hline \multirow[t]{4}{*}{30} & 30 & IVC & DEN2294 & Denmark & 2001 & 1 & ND & ND & {$[39,57]$} \\
\hline & 36 & $\|$ & USA200 & USA & 1995-2003 & 1 & 1 & 1 & {$[40,46]$} \\
\hline & 36 & $\|$ & HAR24 & Finland & 2002 & 1 & ND & ND & {$[46,47]$} \\
\hline & 22 & $\mathrm{IVh}$ & HAR22 & Finland & 2002 & 9 & - & 4 & {$[39,44]$} \\
\hline \multirow[t]{3}{*}{22} & 22 & IVh & HGSA146 & Portugal & 2003 & 9 & ND & ND & {$[39,58]$} \\
\hline & 22 & $\mathrm{IVh}$ & HGSA163 & Portugal & 2003 & 9 & ND & ND & {$[39,58]$} \\
\hline & 45 & $\|$ & USA600 & USA & 1995-2003 & 7 & 4 & 9 & {$[40,46]$} \\
\hline \multirow[t]{3}{*}{45} & 45 & $\mathrm{IVa}$ & HAR38 & Belgium & 1995 & 6 & 2 & 8 & {$[39,44]$} \\
\hline & 45 & V & WIS & Australia & 1995 & 8 & ND & ND & [59] \\
\hline & 256 & $\mathrm{IVa}$ & CA05 & Chicago & 1999 & 8 & 4 & 9 & [45] \\
\hline
\end{tabular}


Table 1 Characteristics and bla locus allotypes of the MRSA strains used in this study (Continued)

\begin{tabular}{cclllllccc}
\hline 1 & 1 & IVa & MW2 & USA & 1998 & 6 & 2 & 10 & {$[60]$} \\
& 1 & IVa & USA400 & USA & $1995-2003$ & 6 & 2 & 10 & {$[39,40]$} \\
\hline 80 & 80 & IVc & DEN2949 & Denmark & 2001 & 5 & 3 & 3 & {$[39,57]$} \\
& 80 & IV & DEN114 & Denmark & 2001 & 5 & 1 & 3 & {$[39,57]$} \\
\hline \multirow{2}{*}{ Singleton } & 377 & $V$ & HT0184 & Greece & 2005 & 6 & 2 & 10 & {$[61]$} \\
& 377 & $V$ & HT0826 & France & 2003 & 6 & ND & ND \\
& & & &
\end{tabular}

a) Clonal complexes were determined using the E-burst software http://saureus.mlst.net/, last accessed on June 04, 2009.

$N D$, not determined, -, negative PCR amplification.

R1, GCA TAT GTT ATT GCT TGA CC; blaI F1, GCA AGT TGA AAT ATC TAT GG; blaI R1, GAA AGG ATC CAT TTT CTG TAC ACT CTC ATC; blaR1 F1, CAT GAC AAT GAA GTA GAA GC; and blaR1 R1, CTT ATG ATT CCA TGA CAT ACG. The predicted amplicon sizes were $533 \mathrm{bp}$ for blaZ, $484 \mathrm{bp}$ for blaI and $537 \mathrm{bp}$ for blaR1. PCR was performed in a T1 Thermocicler (Biometra) with the following conditions: $94^{\circ} \mathrm{C}$ for $4 \mathrm{~min} ; 30$ cycles of $94^{\circ} \mathrm{C}$ for $30 \mathrm{~s}, 55^{\circ} \mathrm{C}$ for $30 \mathrm{~s}$ and $72^{\circ} \mathrm{C}$ for $1 \mathrm{~min}$; and a final extension at $72^{\circ} \mathrm{C}$ for 10 min. In each reaction (final volume of $50 \mu \mathrm{L}$ ), $5 \mathrm{ng}$ of chromosomal template, $2.5 \mathrm{U}$ of GoTaq flexi DNA polymerase (Promega), $1 \times$ Colorless GoTaq flexi buffer (Promega), $2.5 \mathrm{mM} \mathrm{MgCl} 2$ (Promega), $40 \mu \mathrm{M}$ of each deoxynucleoside triphosphate (dNTPs mixture, Bioron) and $20 \mathrm{pmol}$ of the forward and reverse primers were used. The amplified fragments were purified using a mix of Exonuclease and SAP enzymes. Sequencing of both strands was performed by Macrogen http://www.macrogen.com or STAB Vida http://www.stabvida.com.

Table 2 Characteristics and bla locus allotypes of MSSA strains used in this study ${ }^{a)}$

\begin{tabular}{|c|c|c|c|c|c|c|c|c|}
\hline \multirow[t]{2}{*}{ Clonal complex ${ }^{\text {b) }}$} & \multirow[t]{2}{*}{ MLST (ST) } & \multirow[t]{2}{*}{ PFGE type } & \multirow[t]{2}{*}{ Strain } & \multirow[t]{2}{*}{ Origin } & \multirow[t]{2}{*}{ Isolation date } & \multicolumn{3}{|c|}{ bla locus alleles } \\
\hline & & & & & & blaz & blal & blaR1 \\
\hline & 1 & G & IPOP38 & Portugal & 2001 & 6 & 2 & 10 \\
\hline \multirow[t]{2}{*}{1} & 188 & $L$ & IPOP58 & Portugal & 2001 & 6 & 2 & 10 \\
\hline & 573 & M & HSJ109 & Portugal & 1995 & 6 & 2 & 10 \\
\hline \multirow[t]{2}{*}{5} & 5 & $C$ & HSA29 & Portugal & 1992-1993 & 11 & 4 & 7 \\
\hline & 5 & C & IPOP41 & Portugal & 2001 & 6 & 3 & 6 \\
\hline \multirow[t]{2}{*}{8} & 8 & J & IPOP65 & Portugal & 2001 & 8 & 2 & ND \\
\hline & 615 & $\mathrm{E}$ & IPOP32 & Portugal & 2001 & 9 & 1 & 4 \\
\hline 9 & 9 & $\mathrm{D}$ & HSJ122 & Portugal & 1995 & 12 & 1 & 12 \\
\hline 10 & 10 & Q & DCC300 & Portugal & 1996-1997 & 9 & 1 & 5 \\
\hline \multirow[t]{2}{*}{12} & 12 & $x$ & HSJ130 & Portugal & 1995 & 3 & 3 & 6 \\
\hline & 12 & $x$ & Draftees 728 & Portugal & 1996-1997 & 1 & 1 & 1 \\
\hline 15 & 15 & K & HSA9 & Portugal & 1992-1993 & 6 & 9 & ND \\
\hline 20 & 20 & $\mathrm{~N}$ & HSA47 & Portugal & 1992-1993 & 6 & 8 & 11 \\
\hline 22 & 22 & $\mathrm{~T}$ & Draftees 721 & Portugal & 1996-1997 & 6 & 3 & 5 \\
\hline \multirow[t]{2}{*}{25} & 25 & $S$ & HSA76 & Portugal & 1992-1993 & 1 & 1 & 1 \\
\hline & 30 & $A$ & IPOP37 & Portugal & 2001 & 13 & 1 & 1 \\
\hline \multirow[t]{3}{*}{30} & 34 & B & IPOP24 & Portugal & 2001 & 6 & ND & ND \\
\hline & 34 & B & IPOP34 & Portugal & 2001 & 1 & 1 & ND \\
\hline & NA & B & IPOP26 & Portugal & 2001 & 1 & ND & ND \\
\hline \multirow[t]{2}{*}{45} & 45 & $\mathrm{H}$ & HSA19 & Portugal & 1992-1993 & 6 & 2 & 10 \\
\hline & 45 & $\mathrm{H}$ & IPOP56 & Portugal & 2001 & 6 & ND & ND \\
\hline 97 & 97 & $P$ & IPOP50 & Portugal & 2001 & 6 & ND & $\mathrm{ND}$ \\
\hline 121 & 121 & $\mathrm{~F}$ & IPOP44 & Portugal & 2001 & 10 & 1 & 5 \\
\hline Singleton & 580 & $R$ & DCC1185 & Portugal & 1996-1997 & 1 & 1 & 1 \\
\hline
\end{tabular}

a) MSSA strains have been previously characterized by PFGE and MLST [62].

b) Clonal complexes were determined using the E-burst software http://saureus.mlst.net/eburst/database.asp, last accessed on June $04,2009$.

NA, not available; ND, not determined. 


\section{DNA sequences analysis and phylogenetic tree reconstruction}

DNA sequencing raw data analysis and multi-sequence alignments were performed using the DNA Star software package (Lasergene). For the multi-sequence alignments, the Clustal W algorithm was used. In order to maximize sequence reads, raw sequences for bla $Z$ and blaR1 were trimmed immediately after the primer sequences keeping the reading frame. As the reverse primer for blaI (BlaI R1) is located outside of the coding region, the 3' end of the sequence was trimmed at the end of the coding region. For each gene, allotypes were defined taking as reference the extant sequences of the bla locus of Tn552, which were assigned to allotype 1 .

Phylogenetic and molecular evolutionary analyses were conducted using MEGA version 4 [25] and the resultant phylogenetic trees were obtained using the neighbourjoining (NJ) method with bootstrap analysis using 1000 replicates. In order to evaluate the diversity of the bla locus, the Simpson's indexes of diversity (SID) were calculated [26,27] for each locus using the online tool available at http://www.comparingpartitions.info. To estimate selection pressure acting on the bla locus, we computed the $\mathrm{dN} / \mathrm{dS}$ ratios for the three genes. The $\mathrm{dN} / \mathrm{dS}$ ratios were computed for all pairs of alleles with more than $1 \%$ substitutions, in order to give an estimate of the divergence of the alleles while excluding those pairs that, being too similar, would give anomalous $\mathrm{dN}$ / $\mathrm{dS}$ ratios. The $\mathrm{dN} / \mathrm{dS}$ ratios were computed by Model Averaging, as described in [28] and implemented in the KaKs_Calculator application [29]. This approach fits a set of models by maximum likelihood and then computes the weighted average of the models using a second-order Akaike Information Criterion (AICC).

\section{Nucleotide sequence accession numbers}

All nucleotide sequences determined in this study were deposited in Genbank under accession numbers GQ980053-GQ980139 (blaZ alleles), GQ980140GQ980187 (blaI alleles) and GQ980188-GQ980236 (blaR1 alleles).

\section{Results}

The allelic variation in the $\beta$-lactamase locus (bla) was evaluated by sequencing internal fragments of blaZ, blaI and blaR1 genes in a representative collection of international epidemic MRSA clones and also, for comparative purposes, in a diverse collection of MSSA strains.

\section{blaZ allelic variability}

Thirteen different blaZ allotypes were identified within our collection, which comprised 54 MRSA and 24 MSSA (Tables 1 and 2, respectively). Although seven alleles were common to MRSA and MSSA strains, we found four alleles present in MRSA strains only and two present in MSSA strains only. Moreover, the relative frequencies of each allele were different among MRSA and MSSA strains (Table 3); for instance, blaZ allotype 1 was dominant in MRSA strains accounting for 43\% (23 out of 54) of the isolates whereas in MSSA it accounted for $21 \%$ (5 out of 24) of the isolates, and blaZ allotype 6 was present in 11\% (6 out 54) of MRSA but was dominant among MSSA accounting for 46\% (11 out 24) of the isolates. The diversity of blaZ gene as measured by the Simpson index of diversity (SID) was higher for the MRSA collection than for MSSA, although not statistically significant due to the partial overlapping of the confidence intervals (SID $=79.18,95 \%$ CI 69.6-88.8 vs SID $=76.09,95 \%$ CI 61.3-90.9, respectively) - see Table 4. Within the length of blaZ region analyzed (492 nucleotides), we detected 43 unique single-nucleotide polymorphisms (SNP) and on average, each blaZ allele has 12.4 SNP comparing to the prototype blaZ sequence of Tn552 (allele 1) - see Tables 3 and 4. Overall, blaZ alleles were more variable in MSSA than in MRSA (14.7 and 11.4 SNP/allele, respectively). As illustrated by the allelic frequency distribution per MRSA lineage (Figure 1 ) or the cluster tree of the thirteen blaZ alleles found in our collections (Figure 2), there is no clustering according to genetic lineages, as defined by MLST sequence type and SCCmec type, or MSSA/MRSA phenotype; i.e. the same allele could be detected in different genetic lineages or among MRSA and MSSA, and the same lineage could be characterized by several alleles. In addition, there was also no clear clustering of blaZ allotypes according to geographic origin or isolation date of the MRSA isolates (see Table 1).

The BlaZ variability in the MRSA and MSSA strains at the protein level was evaluated by comparison of the deduced amino acid sequence of all alleles against the deduced amino acid sequence for the BlaZ of Tn552. Overall, the deduced amino acid sequences of blaZ alleles from the MRSA and MSSA strains revealed on average 5.8 silent mutations, 1.8 conservative missense mutations and 4 non-conservative missense mutations per allotype (see Tables 3 and 4). For MRSA strain HAR40, a nonsense mutation at Gln76 was detected which presumably originates a non-functional truncated BlaZ protein. As this strain was positive for the nitrocefin test, the DNA extraction and the bla $Z$ sequencing were repeated and the nonsense mutation was confirmed. No frameshift mutations were found in blaZ allotypes.

\section{Allelic variability of blaZ regulatory genes}

Based on the bla $Z$ variability analysis, we selected 51 representative strains to further characterize the variability in the blaZ regulatory genes, blaI and blaR1. Some 
Table 3 Characteristics of bla locus alleles

\begin{tabular}{|c|c|c|c|c|c|c|c|c|}
\hline \multirow[t]{2}{*}{ Gene } & \multirow[t]{2}{*}{ Allele No. } & \multicolumn{2}{|c|}{ Frequency } & \multirow[t]{2}{*}{ SNP $^{c)}$} & \multicolumn{4}{|c|}{ Amino acid substitutions } \\
\hline & & MRSA $^{\text {a) }}$ & MSSA $^{\text {b) }}$ & & Silent & Conservative & Missense & Nonsense \\
\hline \multirow{13}{*}{ blaz } & 1 & 0.43 & 0.21 & 0 & 0 & 0 & 0 & 0 \\
\hline & 2 & 0.02 & 0 & 1 & 0 & 0 & 1 & 1 \\
\hline & 3 & 0.07 & 0.04 & 9 & 4 & 2 & 2 & 0 \\
\hline & 4 & 0.04 & 0 & 9 & 4 & 2 & 3 & 0 \\
\hline & 5 & 0.06 & 0 & 7 & 2 & 2 & 3 & 0 \\
\hline & 6 & 0.11 & 0.46 & 13 & 8 & 2 & 3 & 0 \\
\hline & 7 & 0.02 & 0 & 12 & 6 & 2 & 4 & 0 \\
\hline & 8 & 0.10 & 0.04 & 11 & 6 & 2 & 3 & 0 \\
\hline & 9 & 0.07 & 0.08 & 20 & 9 & 2 & 7 & 0 \\
\hline & 10 & 0.04 & 0.04 & 19 & 8 & 2 & 7 & 0 \\
\hline & 11 & 0.06 & 0.04 & 24 & 11 & 3 & 8 & 0 \\
\hline & 12 & 0 & 0.04 & 24 & 11 & 2 & 8 & 0 \\
\hline & 13 & 0 & 0.04 & 12 & 7 & 2 & 3 & 0 \\
\hline \multirow{9}{*}{ blal } & 1 & 0.33 & 0.45 & 0 & 0 & 0 & 0 & 0 \\
\hline & 2 & 0.15 & 0.25 & 6 & 5 & 0 & 1 & 0 \\
\hline & 3 & 0.19 & 0.15 & 1 & 0 & 0 & 1 & 0 \\
\hline & 4 & 0.19 & 0.05 & 4 & 3 & 0 & 1 & 0 \\
\hline & 5 & 0.04 & 0 & 7 & 5 & 0 & 2 & 0 \\
\hline & 6 & 0.07 & 0 & 4 & 3 & 0 & 1 & 0 \\
\hline & 7 & 0.04 & 0 & 5 & 4 & 0 & 1 & 0 \\
\hline & 8 & 0 & 0.05 & 3 & 1 & 1 & 1 & 0 \\
\hline & 9 & 0 & 0.05 & 1 & 0 & 0 & 1 & 0 \\
\hline \multirow{12}{*}{ blaR1 } & 1 & 0.26 & 0.24 & 0 & 0 & 0 & 0 & 0 \\
\hline & 2 & 0.07 & 0 & 19 & 9 & 4 & 6 & 0 \\
\hline & 3 & 0.10 & 0 & 18 & 7 & 4 & 6 & 0 \\
\hline & 4 & 0.07 & 0.06 & 35 & 15 & 9 & 10 & 0 \\
\hline & 5 & 0.07 & 0.18 & 35 & 15 & 7 & 11 & 0 \\
\hline & 6 & 0.07 & 0.12 & 17 & 6 & 4 & 6 & 0 \\
\hline & 7 & 0.07 & 0.06 & 24 & 10 & 7 & 7 & 0 \\
\hline & 8 & 0.03 & 0 & 33 & 12 & 6 & 12 & 0 \\
\hline & 9 & 0.16 & 0 & 31 & 11 & 6 & 11 & 0 \\
\hline & 10 & 0.13 & 0.24 & 32 & 12 & 6 & 11 & 0 \\
\hline & 11 & 0 & 0.06 & 20 & 9 & 5 & 7 & 0 \\
\hline & 12 & 0 & 0.06 & 34 & 16 & 6 & 10 & 0 \\
\hline
\end{tabular}

a) The total number of MRSA strains whose blaZ, blal and blaR1 genes were analyzed is 54,27 and 31 , respectively.

b) The total number of MSSA strains whose blaZ, blal and blaR1 genes were analyzed is 24, 20 and 17, respectively.

c) For each allele, the SNP were counted taking as reference Tn552 bla sequences (allele 1).

of these strains failed in the amplification of one of the bla $Z$ regulatory genes (see Tables 1 and 2).

Within the length of blaI region analyzed (351 nucleotides), we detected 13 unique SNP, which account for the nine blaI allotypes detected (see Tables 3 and 4). Four of the nine blaI allotypes were present in both MRSA and MSSA, while three blaI allotypes were found in MRSA strains only and two in MSSA only. The SID was higher for MRSA than for MSSA although not statistically significant (SID $=82.1,95 \%$ CI 74.6-89.5 vs SID $=74.2$, 95\% CI 60.5-87.9, respectively) (Table 4). On average, each blaI allele has 3.4 SNP comparing to the prototype blaI sequence of Tn552 (allele 1), and blaI alleles were on average more polymorphic for MRSA than for MSSA (3.9 vs 2.5 SNP per allele, respectively) see Tables 3 and 4 .

Within the length of blaR1 region analyzed (498 nucleotides), we detected 65 unique SNP, which account for the 12 blaR 1 allotypes detected (see Tables 3 and 4). Six of the 12 blaR 1 allotypes were present in both MRSA and MSSA, while four blaR1 allotypes were unique for MRSA strains and two were characteristic of MSSA strains. The SID values were virtually identical for both MRSA and MSSA (SID = 88.8, 95\%CI 83.2- 
Table 4 Comparative analysis of the allelic variation in bla locus for MRSA and MSSA strains

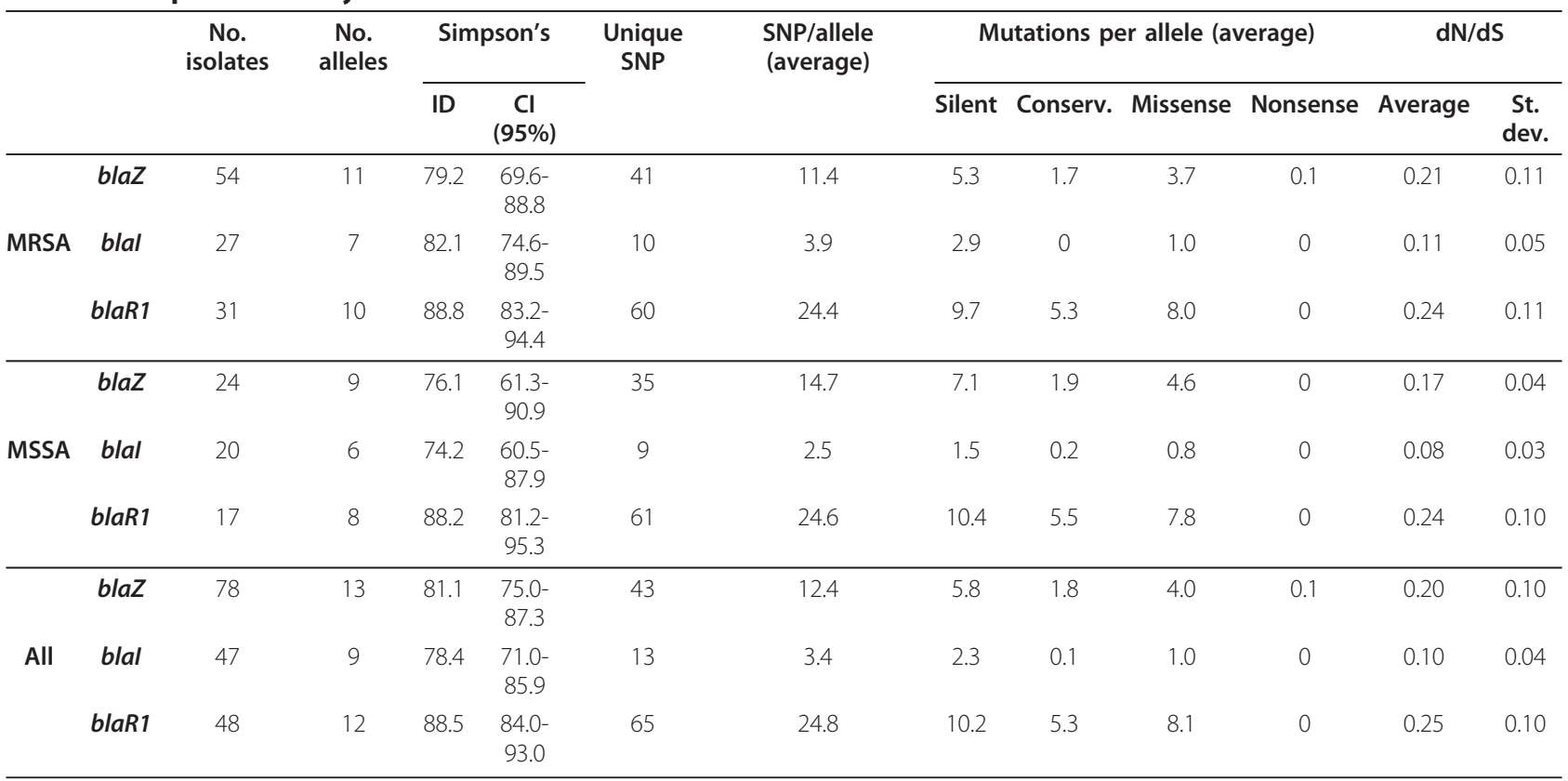

ID, index of diversity; $\mathrm{Cl}$, confidence interval; SNP, single-nucleotide polymorphism; Conserv., conservative; St. dev., standard deviation

94.4 vs SID $=88.2$, 95\%CI 81.2-95.3, respectively) (Table 4). On average, each blaR1 allele has 24.8 SNP comparing to the prototype blaR1 sequence of Tn552 (allele 1), with no significant differences between MRSA and MSSA (24.4 and 24.6 SNP/allele, respectively) - see Tables 3 and 4.

In agreement with what was observed for the blaZ gene, the cluster trees of blaI and blaR1 alleles found in our collections also showed no clustering according to MSSA/MRSA phenotype or genetic lineages (Figures 3 and 4). For those strains in which the alleles of the three genes were determined, we constructed a cluster tree with the concatenated sequences - see Figure 5. In spite

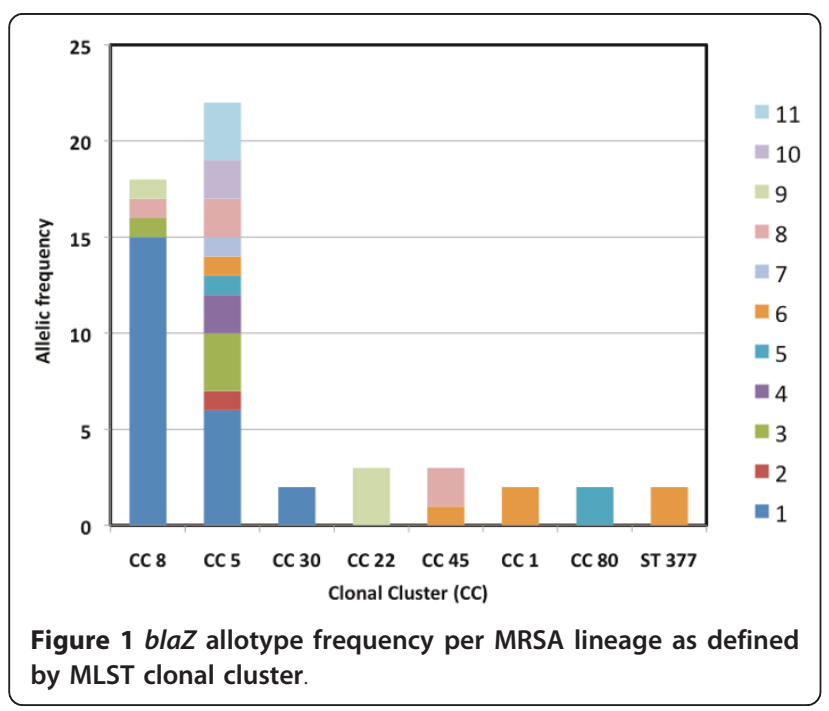

of the relatively low number of allelic profiles, there was still no clear clustering of bla allotypes according to MSSA/MRSA phenotype or lineage, as the same allelic profile was present in different genetic lineages (e.g. profile $8 / 4 / 9$ present in clonal complexes 5, 8 and 45) and, the same genetic lineage was characterized by profiles from different brunches (e.g. clonal cluster 8 characterized by profiles $8 / 4 / 9,1 / 1 / 1,3 / 3 / 6$, etc.).

The BlaI and BlaR1 variabilities at the protein level in the MRSA and MSSA strains were evaluated by comparison of the deduced amino acid sequence of all alleles against the corresponding deduced amino acid sequences of $\operatorname{Tn} 552$ (see Tables 3 and 4).

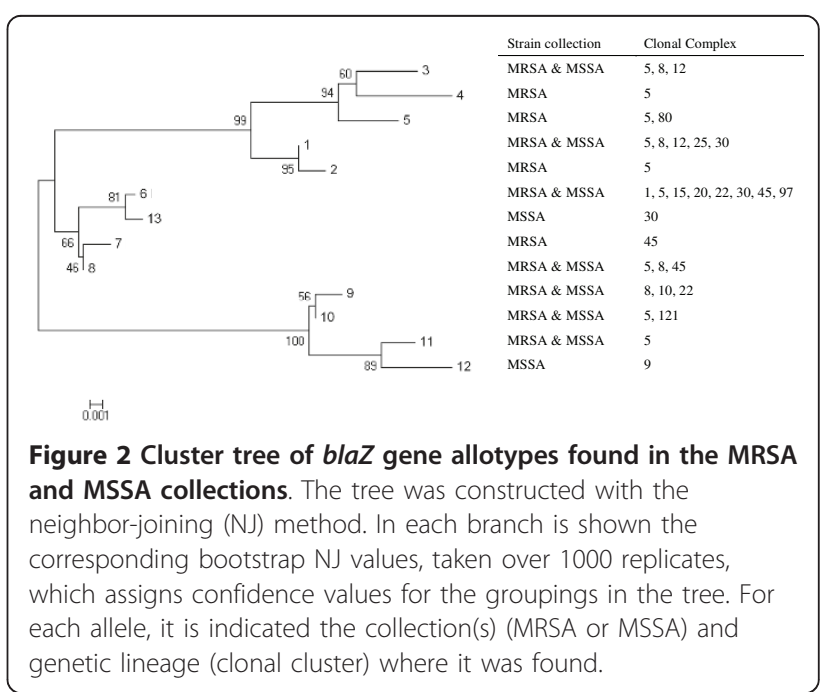




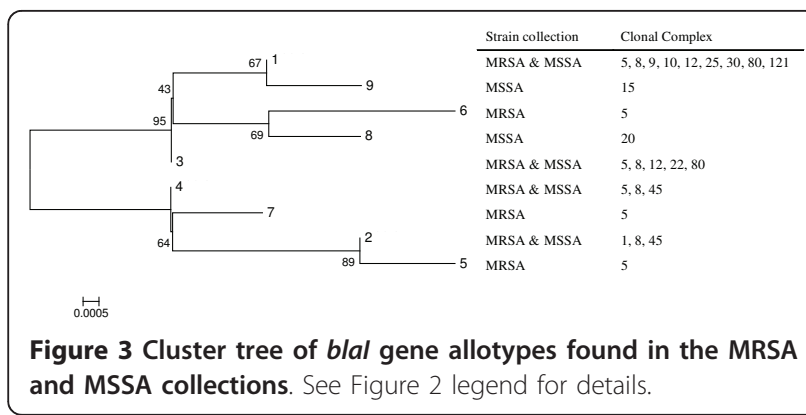

Overall, the deduced amino acid sequences of the blaI alleles revealed on average 2.3 silent mutations, 0.1 conservative missense mutations and 1 non-conservative missense mutation per allotype. The deduced amino acid sequences of the blaR1 alleles showed on average 10.2 silent mutations, 5.3 conservative missense mutations and 8.1 non-conservative missense mutations per allotype. None of the SNP detected within the blaI or blaR1 resulted in nonsense or frameshift mutations.

\section{Selection pressure acting on the bla locus}

Based on the allelic data obtained, we computed the $\mathrm{dN} /$ $\mathrm{dS}$ ratios as estimates for the selective pressure acting on the bla locus. The $\mathrm{dN} / \mathrm{dS}$ ratios were computed for all pairs of alleles differing more than $1 \%$, in order to give an estimate of the allelic divergence, excluding the anomalous $\mathrm{dN} / \mathrm{dS}$ ratios of those pairs being very similar. The average of the obtained $\mathrm{dN} / \mathrm{dS}$ values and respective standard deviations are summarized in Table 4. The dN/dS values for the three genes in the MRSA, MSSA and MRSA/MSSA partitions were well below 1 (between 0.08 and 0.25 with standard deviations between 0.05 and 0.1 ), which suggests a negative or purifying selection acting on the bla locus. In agreement with the average number of SNP per allele, the $\mathrm{dN} / \mathrm{dS}$ ratios were significantly higher for the blaR1 gene $(0.24$ - 0.25) and lower for mecI (0.08 - 0.11).

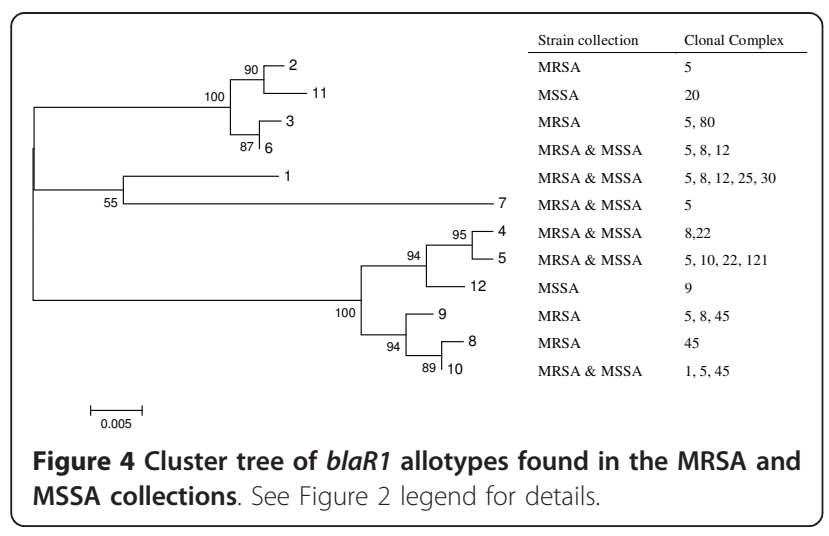

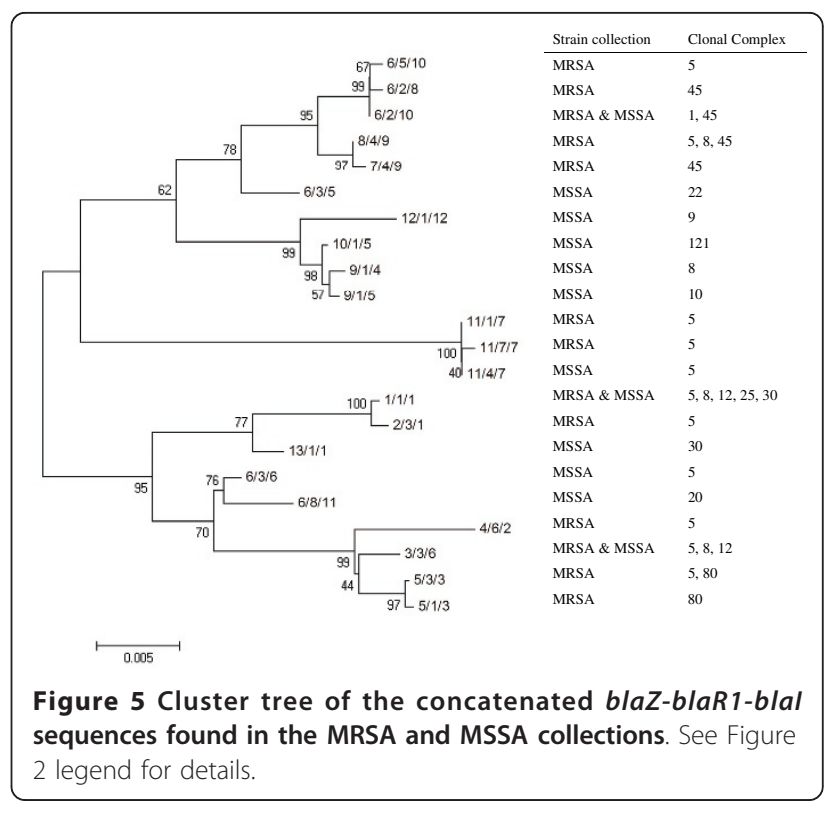

\section{Discussion}

The rationale for this study comes from several observations strongly suggesting a role of bla genes in the acquisition, stabilization and regulation of $m e c A$ gene, the central element of "broad-spectrum" $\beta$-lactam resistance characteristic of MRSA strains. The purpose of this study was to evaluate the allelic variability of the bla locus in a representative collection of international epidemic MRSA clones and also, for comparative purposes, in a diverse collection of MSSA strains, in an attempt to establish evolutionary correlations between bla allotypes and $\beta$-lactam resistance phenotypes (i.e. between MRSA and MSSA), SCCmec types (i.e. polymorphisms in the mecA regulatory locus) and/or genetic lineages.

MRSA lineages are much less diverse than MSSA lineages in terms of their genome content, a consequence of their more recent evolutionary history $[19,20]$ and, apparently, also due to some "host barrier" to the SCCmec acquisition [13]. These differences in genetic background variability were well illustrated in our collections since the international MRSA collection comprised eight lineages as defined by MLST clonal complexes, whereas in the smaller and local MSSA collection 15 lineages were represented.

In contrast to the genetic background diversity, we could not detect significant differences between MSSA and MRSA in terms of the bla locus allelic variability. Actually, there were disparate subtle differences in terms of number of allotypes and number of point mutations per allotype: e.g. 11 vs 9 blaZ allotypes and 11.4 vs 14.7 SNP/allele in MRSA and MSSA, respectively. These subtle differences may reflect the more 
ancient evolutionary history of MSSA or a selective pressure to improve the bla locus activity in these strains. That is to say, although fewer bla types have been retained by the natural selection in MSSA, on average, these allotypes seem to have accumulated more adaptive mutations, in comparison to MRSA strains. In particular for blaZ, for which differences in terms of number of alleles and SNP/allele were more significant, the presence of the alternative $\beta$-lactam resistance mechanism mediated by the mecA gene in MRSA strains might have allowed a release in the selective pressure to keep bla $Z$ with optimal activity, in contrast to MSSA, which rely only on blaZ-mediated resistance to $\beta$-lactams.

No correlation could be established between bla allotypes and strain backgrounds, $\beta$-lactam resistance phenotypes, strain origin and/or isolation dates, indicating that bla genes have evolved independently from $S$. aureus clonal lineages. This is particularly striking for MRSA strains, which have a very strong clonal structure. These observations may be explained either by differences in evolutionary clock speeds between the genetic background and the bla locus or may result from the horizontal transfer of bla genes between different lineages, which are usually integrated in mobile elements (plasmids and composite transposons). Interestingly, based on the characterization of a collection of several staphylococcal species, Olsen et al, suggested that there is little exchange of bla genes between strains or species [14], which somehow contradicts our findings. In our study, the most parsimony explanation for the presence of the same bla type in different genetic lineages either MRSA or MSSA or the presence of several bla types in the same lineage, is indeed a high frequency for the horizontal transfer of bla genes across $S$. aureus clonal clusters.

In spite of the lack of evolutionary links between bla allotypes and genetic lineages, our data strongly suggests a selective pressure to keep the bla locus fully functional, as illustrated by the calculated average $\mathrm{dN} / \mathrm{dS}$ values well below 1 . This observation is valid even on MRSA for which one could expect the accumulation of nonsense or frameshift mutations that would render the bla locus non-functional, due to presence of the $m e c A$ gene. Actually, the majority of the mutational events detected in this study were either silent or neutral mutations, being the blaR1 the gene with the highest mutational rate and the blaI the one with the lowest. The increased allelic variability detected for blaR1 (in terms of number of alleles, Simpson's index of diversity, average $\mathrm{SNP} /$ allele, and $\mathrm{dN} / \mathrm{dS}$ values) may suggest that this sensor-inducer gene is the primary target for the evolutionary adaptive mechanisms in the bla locus, presumably to improve the induction efficiency of blaZ expression or even $m e c A$ expression, in the case of MRSA strains with no functional $m e c I-m e c R 1$ regulatory system. In contrast, the relatively lower variability of the much smaller blaI gene, may suggest a fine-tuned repressor activity and a selective pressure to maintain the repressor activity; i.e to maintain the blaZ expression inducible.

Despite the cross-resistance to virtually all $\beta$-lactam antibiotics provided by mecA, most contemporary MRSA strains still carry, besides the SCCmec element, the $\beta$-lactamase locus. This might be due to the fact that not enough time has elapsed since the mecA acquisition for MRSA strains start loosing the bla genes, because there is a little or no fitness cost associated to the bla genes, or because these genes may be linked to other positively selected genes (e.g. the cadmium resistance genes present in some $\beta$-lactamase plasmids). Alternatively, the bla locus may be involved in the "domestication" of the mecA gene, as bla genes have been shown to stabilize the in vitro mec $A$ acquisition $[12,13]$ and efficiently control mecA transcription $[9,10]$, explaining the "retention" of a functional bla regulatory system by most contemporary MRSA strains [8]. Interestingly, as no correlation could be established between bla allotypes and SCCmec types, which have polymorphisms in the mecA regulatory locus, this maintenance of functional blaI-blaR1 genes seems to be independent of the functional status of the mecA "natural" regulators mecI-mecR1.

Concerning the maintenance of a functional bla $Z$ gene in MRSA strains one can speculate that, even in the presence of $m e c A$, it might be useful for the bacteria to keep bla $Z$ as a "first-line defense" against $\beta$-lactams. In fact, first generation $\beta$-lactams (i.e. penicillins) are still widely prescribed either empirically or for the treatment of specific infections (e.g. streptococcal infections). Moreover, penicillins have also been widely used prophylactically in the livestock industry. This means that, both in the nosocomial and community settings, MRSA are still exposed to penicillins and, under these circumstances, expression of $\beta$-lactamase is enough for survival under antibiotic pressure. From a physiological perspective, this ability to choose between the expression of two resistance genes may be advantageous for the bacteria since the expression of $\beta$-lactamase is likely to impose a smaller fitness cost than the expression of PBP2a. In fact, besides being much smaller than PBP2a (257 vs 668 amino acids), BlaZ is a secreted enzyme whereas PBP2a is a transpeptidase protein, which must be incorporated into the complex cell-wall metabolism.

\section{Conclusion}

In this study we have evaluated the allelic variation of the bla locus in MRSA and MSSA clinical strains. Although 
no correlation between bla allotypes and genetic lineages, SCCmec types and $\beta$-lactam resistance phenotypes could be established, we provided evidence for the existence of a selective pressure to maintain the bla system fully functional even on MRSA strains and that the sensor-inducer gene blaR 1 is the primary target for the accumulation of adaptive mutations in the bla locus.

\section{Acknowledgements}

We thank T. Ito, D.C. Coleman, R. Daum, K.T. Park, W.B. Grubb, and A. Tomasz for having kindly given some of the prototype and reference strains used in this study. We thank J. Almeida for the assistance on the numerical data analysis.

Partial support for this study was provided by Projects POCI/BIA-MIC/60320/ 2004 and PTDC/BIA-MIC/64071/2006 from Fundação para a Ciência e Tecnologia (FCT), Lisbon, Portugal awarded to D.C. Oliveira and Project TROCAR, Contract number HEALTH-F3-2008-223031 from the European Commission awarded to $\mathrm{H}$. de Lencastre. C. Milheiriço was supported by grant SFRH/BPD/63992/2009 from FCT.

\section{Author details \\ ${ }^{1}$ Laboratory of Molecular Genetics, Instituto de Tecnologia Química e Biológica, Universidade Nova de Lisboa (ITQB/UNL), Oeiras, Portugal. ${ }^{2}$ CREM, Department of Life Sciences, Faculdade de Ciências e Tecnologia, Universidade Nova de Lisboa (FCT/UNL), Caparica, Portugal. ${ }^{3}$ CENTRIA, Department of Informatics, Faculdade de Ciências e Tecnologia, Universidade Nova de Lisboa. ${ }^{4}$ Laboratory of Microbiology, The Rockefeller University, New York, NY, USA.}

\section{Authors' contributions}

CM participated in the study design, carried out experimental work, analyzed and interpreted data and wrote the manuscript. AP carried out experimental work and analyzed data. LK analyzed and interpreted data. HdL participated in study design and corrected the manuscript. DCO conceived the study, participated in the study design, interpreted the data and wrote the manuscript. All authors have read and approved the manuscript.

\section{Competing interests}

The authors declare that they have no competing interests.

Received: 4 January 2011 Accepted: 15 April 2011

Published: 15 April 2011

\section{References}

1. Klevens RM, Morrison MA, Nadle J, Petit S, Gershman K, Ray S, Harrison LH, Lynfield R, Dumyati G, Townes JM, et al: Invasive methicillin-resistant Staphylococcus aureus infections in the United States. Jama 2007, 298(15):1763-1771.

2. Chambers HF: The changing epidemiology of Staphylococcus aureus? Emerg Infect Dis 2001, 7(2):178-182.

3. Furuya EY, Lowy FD: Antimicrobial-resistant bacteria in the community setting. Nat Rev Microbiol 2006, 4(1):36-45.

4. de Lencastre $H$, Oliveira D, Tomasz A: Antibiotic resistant Staphylococcus aureus: a paradigm of adaptive power. Curr Opin Microbiol 2007, 10(5):428-435.

5. Wilke MS, Lovering AL, Strynadka NC: Beta-lactam antibiotic resistance: a current structural perspective. Curr Opin Microbiol 2005, 8(5):525-533.

6. Barber M, Rozwadowska-Dowzenko M: Infection by penicillin-resistant staphylococci. Lancet 1948, 2(6530):641-644.

7. Hartman B, Tomasz A: Altered penicillin-binding proteins in methicillinresistant strains of Staphylococcus aureus. Antimicrob Agents Chemother 1981, 19(5):726-735.

8. Livermore DM: Beta-Lactamases in Laboratory and Clinical Resistance. Clin Microbiol Rev 1995, 8(4):557-584.

9. Hackbarth CJ, Chambers HF: blal and blaR1 regulate beta-lactamase and PBP2a production in methicillin-resistant Staphylococcus aureus. Antimicrob Agents Chemother 1993, 37(5):1144-1149.
10. Ryffel C, Kayser FH, Berger-Bachi B: Correlation between regulation of mecA transcription and expression of methicillin resistance in staphylococci. Antimicrob Agents Chemother 1992, 36(1):25-31.

11. International Working Group on the Classification of Staphylococcal Cassette Chromosome Elements (IWG-SCC): Classification of staphylococcal cassette chromosome mec (SCCmec): guidelines for reporting novel SCCmec elements. Antimicrob Agents Chemother 2009, 53(12):4961-4967.

12. Cohen S, Sweeney HM: Effect of the prophage and penicillinase plasmid of the recipient strain upon the transduction and the stability of methicillin resistance in Staphylococcus aureus. J Bacteriol 1973, 116(2):803-811.

13. Katayama $Y$, Zhang HZ, Hong D, Chambers HF: Jumping the barrier to beta-lactam resistance in Staphylococcus aureus. J Bacteriol 2003, 185(18):5465-5472.

14. Olsen JE, Christensen H, Aarestrup FM: Diversity and evolution of blaZ from Staphylococcus aureus and coagulase-negative staphylococci. J Antimicrob Chemother 2006, 57(3):450-460.

15. Ambler RP: The structure of beta-lactamases. Philos Trans $R$ Soc Lond $B$ Biol Sci 1980, 289(1036):321-331.

16. Richmond $\mathrm{MH}$ : Wild-Type Variants of Exopenicillinase from Staphylococcus aureus. Biochem J 1965, 94:584-593.

17. East AK, Dyke KG: Cloning and sequence determination of six Staphylococcus aureus beta-lactamases and their expression in Escherichia coli and Staphylococcus aureus. J Gen Microbiol 1989, 135(4):1001-1015.

18. Ito $T$, Katayama $Y$, Hiramatsu $K$ : Cloning and nucleotide sequence determination of the entire mec DNA of pre-methicillin-resistant Staphylococcus aureus N315. Antimicrob Agents Chemother 1999, 43(6):1449-1458

19. Enright MC, Robinson DA, Randle G, Feil EJ, Grundmann H, Spratt BG: The evolutionary history of methicillin-resistant Staphylococcus aureus (MRSA). Proc Natl Acad Sci USA 2002, 99(11):7687-7692.

20. Oliveira DC, Tomasz A, de Lencastre H: Secrets of success of a human pathogen: molecular evolution of pandemic clones of meticillin-resistant Staphylococcus aureus. Lancet Infect Dis 2002, 2(3):180-189.

21. Katayama Y, Robinson DA, Enright MC, Chambers HF: Genetic background affects stability of mecA in Staphylococcus aureus. J Clin Microbiol 2005, 43(5):2380-2383

22. Nubel U, Roumagnac P, Feldkamp M, Song JH, Ko KS, Huang YC, Coombs G, Ip M, Westh H, Skov R, et al: Frequent emergence and limited geographic dispersal of methicillin-resistant Staphylococcus aureus. Proc Natl Acad Sci USA 2008, 105(37):14130-14135.

23. Smyth DS, McDougal LK, Gran FW, Manoharan A, Enright MC, Song JH, de Lencastre $\mathrm{H}$, Robinson DA: Population structure of a hybrid clonal group of methicillin-resistant Staphylococcus aureus, ST239-MRSA-III. PLOS One 2010, 5(1):e8582.

24. Harris SR, Feil EJ, Holden MT, Quail MA, Nickerson EK, Chantratita N, Gardete S, Tavares A, Day N, Lindsay JA, et al: Evolution of MRSA during hospital transmission and intercontinental spread. Science 2010, 327(5964):469-474.

25. Tamura K, Dudley J, Nei M, Kumar S: MEGA4: Molecular Evolutionary Genetics Analysis (MEGA) software version 4.0. Mol Biol Evol 2007, 24(8):1596-1599.

26. Grundmann H, Hori S, Tanner G: Determining confidence intervals when measuring genetic diversity and the discriminatory abilities of typing methods for microorganisms. J Clin Microbiol 2001, 39(11):4190-4192.

27. Simpson EH: Measurement of diversity. Nature 1949, 163:688-688

28. Posada D, Buckley TR: Model selection and model averaging in phylogenetics: advantages of akaike information criterion and bayesian approaches over likelihood ratio tests. Syst Biol 2004, 53(5):793-808.

29. Zhang Z, Li J, Zhao XQ, Wang J, Wong GK, YU J: KaKs_Calculator: calculating $\mathrm{Ka}$ and $\mathrm{Ks}$ through model selection and model averaging. Genomics Proteomics Bioinformatics 2006, 4(4):259-263.

30. Oliveira DC, Tomasz A, de Lencastre $\mathrm{H}$ : The evolution of pandemic clones of methicillin-resistant Staphylococcus aureus: identification of two ancestral genetic backgrounds and the associated mec elements. Microb Drug Resist 2001, 7(4):349-361.

31. de Lencastre $H$, Chung $M$, Westh $H$ : Archaic strains of methicillin-resistant Staphylococcus aureus: molecular and microbiological properties of isolates from the 1960s in Denmark. Microb Drug Resist 2000, 6(1):1-10. 
32. Sanches IS, Ramirez M, Troni H, Abecassis M, Padua M, Tomasz A, de Lencastre $\mathrm{H}$ : Evidence for the geographic spread of a methicillin-resistant Staphylococcus aureus clone between Portugal and Spain. J Clin Microbiol 1995, 33(5):1243-1246.

33. Roberts RB, Tennenberg AM, Eisner W, Hargrave J, Drusin LM, Yurt R, Kreiswirth BN: Outbreak in a New York City teaching hospital burn center caused by the Iberian epidemic clone of MRSA. Microb Drug Resist 1998, 4(3):175-183.

34. Kreiswirth B, Kornblum J, Arbeit RD, Eisner W, Maslow JN, McGeer A, Low DE, Novick RP: Evidence for a clonal origin of methicillin resistance in Staphylococcus aureus. Science 1993, 259(5092):227-230.

35. Dominguez MA, de Lencastre $H$, Linares J, Tomasz A: Spread and maintenance of a dominant methicillin-resistant Staphylococcus aureus (MRSA) clone during an outbreak of MRSA disease in a Spanish hospital. J Clin Microbiol 1994, 32(9):2081-2087.

36. Dubin DT, Chikramane SG, Inglis B, Matthews PR, Stewart PR: Physical mapping of the mec region of an Australian methicillin-resistant Staphylococcus aureus lineage and a closely related American strain. J Gen Microbiol 1992, 138(3):657.

37. Teixeira LA, Resende CA, Ormonde LR, Rosenbaum R, Figueiredo AM, de Lencastre H, Tomasz A: Geographic spread of epidemic multiresistant Staphylococcus aureus clone in Brazil. J Clin Microbiol 1995, 33(9):2400-2404.

38. de Lencastre $H$, Severina EP, Milch $H$, Thege MK, Tomasz A: Wide geographic distribution of a unique methicillin-resistant Staphylococcus aureus clone in Hungarian hospitals. Clin Microbiol Infect 1997 3(3):289-296.

39. Milheirico C, Oliveira DC, de Lencastre H: Multiplex PCR strategy for subtyping the staphylococcal cassette chromosome mec type IV in methicillin-resistant Staphylococcus aureus: 'SCCmec IV multiplex'. J Antimicrob Chemother 2007, 60(1):42-48.

40. McDougal LK, Steward CD, Killgore GE, Chaitram JM, McAllister SK, Tenover FC: Pulsed-field gel electrophoresis typing of oxacillin-resistant Staphylococcus aureus isolates from the United States: establishing a national database. J Clin Microbiol 2003, 41(11):5113-5120.

41. Roberts RB, de Lencastre A, Eisner W, Severina EP, Shopsin B, Kreiswirth BN, Tomasz A: Molecular epidemiology of methicillin-resistant Staphylococcus aureus in 12 New York hospitals. MRSA Collaborative Study Group. $J$ Infect Dis 1998, 178(1):164-171.

42. Shore A, Rossney AS, Keane CT, Enright MC, Coleman DC: Seven novel variants of the staphylococcal chromosomal cassette mec in methicillinresistant Staphylococcus aureus isolates from Ireland. Antimicrob Agents Chemother 2005, 49(5):2070-2083.

43. Aires de Sousa M, de Lencastre H: Evolution of sporadic isolates of methicillin-resistant Staphylococcus aureus (MRSA) in hospitals and their similarities to isolates of community-acquired MRSA. J Clin Microbiol 2003, 41(8):3806-3815

44. Cookson B, HARMONY participants: HARMONY - The International Union of Microbiology Societies' European Staphylococcal Typing Network. Eurosurveillance 2008, 13(19):Article 4 [http://www.eurosurveillance.org/ ViewArticle.aspx?Articleld=18860)],

45. Ma XX, Ito T, Tiensasitorn C, Jamklang M, Chongtrakool P, Boyle-Vavra S, Daum RS, Hiramatsu K: Novel type of staphylococcal cassette chromosome mec identified in community-acquired methicillin-resistant Staphylococcus aureus strains. Antimicrob Agents Chemother 2002, 46(4):1147-1152.

46. Milheirico C, Oliveira DC, de Lencastre H: Update to the multiplex PCR strategy for assignment of mec element types in Staphylococcus aureus. Antimicrob Agents Chemother 2007, 51(9):3374-3377.

47. Oliveira DC, Milheirico C, Vinga S, de Lencastre $\mathrm{H}$ : Assessment of allelic variation in the $c C r A B$ locus in methicillin-resistant Staphylococcus aureus clones. J Antimicrob Chemother 2006, 58(1):23-30.

48. Aires de Sousa M, de Lencastre H, Santos Sanches I, Kikuchi K, Totsuka K, Tomasz A: Similarity of antibiotic resistance patterns and molecular typing properties of methicillin-resistant Staphylococcus aureus isolates widely spread in hospitals in New York City and in a hospital in Tokyo, Japan. Microb Drug Resist 2000, 6(3):253-258.

49. de Lencastre $H$, Severina EP, Roberts RB, Kreiswirth BN, Tomasz A: Testing the efficacy of a molecular surveillance network: methicillin-resistant Staphylococcus aureus (MRSA) and vancomycin-resistant Enterococcus faecium (VREF) genotypes in six hospitals in the metropolitan New York
City area. The BARG Initiative Pilot Study Group. Bacterial Antibiotic Resistance Group. Microb Drug Resist 1996, 2(3):343-351.

50. de Lencastre $H$, de Lencastre A, Tomasz A: Methicillin-resistant Staphylococcus aureus isolates recovered from a New York City hospital: analysis by molecular fingerprinting techniques. J Clin Microbiol 1996, 34(9):2121-2124.

51. Sa-Leao R, Santos Sanches I, Dias D, Peres I, Barros RM, de Lencastre H: Detection of an archaic clone of Staphylococcus aureus with low-level resistance to methicillin in a pediatric hospital in Portugal and in international samples: relics of a formerly widely disseminated strain? J Clin Microbiol 1999, 37(6):1913-1920.

52. Adcock PM, Pastor P, Medley F, Patterson JE, Murphy TV: Methicillinresistant Staphylococcus aureus in two child care centers. J Infect Dis 1998, 178(2):577-580

53. Ma XX, Ito T, Chongtrakool P, Hiramatsu K: Predominance of clones carrying Panton-Valentine leukocidin genes among methicillin-resistant Staphylococcus aureus strains isolated in Japanese hospitals from 1979 to 1985. J Clin Microbiol 2006, 44(12):4515-4527.

54. Kwon NH, Park KT, Moon JS, Jung WK, Kim SH, Kim JM, Hong SK, Koo HC, Joo YS, Park YH: Staphylococcal cassette chromosome mec (SCCmec) characterization and molecular analysis for methicillin-resistant Staphylococcus aureus and novel SCCmec subtype IVg isolated from bovine milk in Korea. J Antimicrob Chemother 2005, 56(4):624-632.

55. Gomes AR, Sanches IS, Aires de Sousa M, Castaneda E, de Lencastre H: Molecular epidemiology of methicillin-resistant Staphylococcus aureus in Colombian hospitals: dominance of a single unique multidrug-resistant clone. Microb Drug Resist 2001, 7(1):23-32.

56. Oliveira DC, Milheirico C, de Lencastre H: Redefining a structural variant of staphylococcal cassette chromosome mec, SCCmec type VI. Antimicrob Agents Chemother 2006, 50(10):3457-3459.

57. Faria NA, Oliveira DC, Westh H, Monnet DL, Larsen AR, Skov R, de Lencastre $\mathrm{H}$ : Epidemiology of emerging methicillin-resistant Staphylococcus aureus (MRSA) in Denmark: a nationwide study in a country with low prevalence of MRSA infection. J Clin Microbiol 2005, 43(4):1836-1842.

58. Amorim ML, Faria NA, Oliveira DC, Vasconcelos C, Cabeda JC, Mendes AC, Calado E, Castro AP, Ramos MH, Amorim JM, et al: Changes in the clonal nature and antibiotic resistance profiles of methicillin-resistant Staphylococcus aureus isolates associated with spread of the EMRSA-15 clone in a tertiary care Portuguese hospital. J Clin Microbiol 2007, 45(9):2881-2888.

59. Ito T, Ma XX, Takeuchi F, Okuma K, Yuzawa H, Hiramatsu K: Novel type V staphylococcal cassette chromosome mec driven by a novel cassette chromosome recombinase, ccrC. Antimicrob Agents Chemother 2004, 48(7):2637-2651

60. Baba T, Takeuchi F, Kuroda M, Yuzawa H, Aoki K, Oguchi A, Nagai Y, Iwama N, Asano K, Naimi T, et al: Genome and virulence determinants of high virulence community-acquired MRSA. Lancet 2002, 359(9320):1819-1827.

61. Tristan A, Bes M, Meugnier H, Lina G, Bozdogan B, Courvalin P, Reverdy ME, Enright MC, Vandenesch F, Etienne J: Global distribution of PantonValentine leukocidin-positive methicillin-resistant Staphylococcus aureus, 2006. Emerg Infect Dis 2007, 13(4):594-600.

62. Aires de Sousa M, Conceicao T, Simas C, de Lencastre H: Comparison of genetic backgrounds of methicillin-resistant and -susceptible Staphylococcus aureus isolates from Portuguese hospitals and the community. J Clin Microbiol 2005, 43(10):5150-5157.

doi:10.1186/1471-2180-11-76

Cite this article as: Milheiriço et al.: Evidence for a purifying selection acting on the $\beta$-lactamase locus in epidemic clones of methicillinresistant Staphylococcus aureus. BMC Microbiology 2011 11:76. 